Décadrages Décadrages

cinéma, à travers champs Cinéma, à travers champs

29-30 | 2015

René Vautier

\title{
La Folle de Toujane : parler ou « faire quelque chose »?
}

\section{Sylvain Dreyer}

\section{Q OpenEdition}

1 Journals

\section{Édition électronique}

URL : https://journals.openedition.org/decadrages/798

DOI : $10.4000 /$ decadrages.798

ISSN : 2297-5977

Éditeur

Association Décadrages

Édition imprimée

Date de publication : 1 avril 2015

Pagination : 87-101

ISBN : 9782970096306

ISSN : 2235-7823

\section{Référence électronique}

Sylvain Dreyer, "La Folle de Toujane : parler ou « faire quelque chose » ? », Décadrages [En ligne], 29-30 2015, mis en ligne le 01 mars 2017, consulté le 25 mars 2023. URL : http://journals.openedition.org/ decadrages/798; DOI : https://doi.org/10.4000/decadrages.798

\section{(c) (i) (2) (2)}

Creative Commons - Attribution - Pas d'Utilisation Commerciale - Partage dans les Mêmes Conditions 4.0 International - CC BY-NC-SA 4.0

https://creativecommons.org/licenses/by-nc-sa/4.0/ 
Sylvain Dreyer

\section{La Folle de Toujane: parler ou « faire quelque chose »?}

La Folle de Toujane ou comment on devient un ennemi de l'intérieur (I974), coréalisé avec Nicole Le Garrec, apparaît dans la carrière de Vautier comme un film de synthèse. Il permet en effet de réunir les deux principaux combats cinématographiques de Vautier: la guerre d'Algérie - depuis Une nation, l'Algérie (I954), avec notamment Algérie en flammes tourné dans les maquis de l'ALN en 1956-1957 - et les luttes politiques en Bretagne - à partir de Mourir pour des images en I97I. L'histoire est racontée en voix over par un instituteur: deux de ses anciens élèves, Roger (Gilles Servat) et Gwen (Micheline Welter), sont amoureux, mais la vie va les séparer. Lui devient instituteur à Toujane en Tunisie, prend conscience de l'oppression coloniale et rejoint le maquis algérien. Elle poursuit une carrière de journaliste à Paris, acceptant toutes les compromissions.

\section{Les documents fictifs de René Vautier}

La Folle de Toujane, tourné deux ans après Avoir 20 ans dans les Aurès (I972), constitue l'aboutissement d'une recherche de formes s'écartant du cinéma documentaire et confinant à la mise en scène de fiction. Dès 1956, René Vautier tourne en Tunisie deux courts métrages: Plages tunisiennes et Les Anneaux d'or. Après le scénario pour le film Des goûts et des couleurs (1969), il réalise en I970 un ensemble de quatre courts métrages en r6mm qui semblent former deux diptyques. Les Trois Cousins (20 min) et Les Ajoncs (Io min) abordent les conditions de vie des travailleurs algériens immigrés en France. L'image de ces deux films est signée Bruno Muel et Robert Lézian, et le rôle principal est confié à Mohamed Zinet ${ }^{1}$ qui, par son interprétation à la fois triste et enjouée, évoque le comique absurde d'un Buster Keaton et plus certainement d'un Chaplin. En effet, lorsque Vautier expose son projet de film dénonçant le sort des immigrés algériens à ses acteurs, ceux-ci lui répondent: «Ça ne sert à rien de faire pleurer les gens; au contraire, si on peut les faire rire avec nos ennuis, c'est mieux. Comme faisait Charlot!» ${ }^{2}$ Le second diptyque
1 Ancien maquisard, puis comédien et assistant réalisateur sur La Bataille d'Alger (i966) de Gillo Pontecorvo.

2 René Vautier, Caméra citoyenne: Mémoires, Rennes, Apogée, I998, p. 213. 
3 Le «cinéma direct» ou «cinéma vérité» est une forme du cinéma documentaire permise par l'arrivée sur le marché de caméras légères et compactes à la fin des années i950. Les exemples les plus connus en France de ce type de cinéma sont Chronique d'un été (I96I) de Jean Rouch et Edgar Morin et Le Joli Mai (I962) de Chris Marker. Voir Gilles Marsolais, L'Aventure du cinéma direct revisitée, Laval (Québec), Les 400 coups, 1997.

4 Roger Odin, «Film documentaire, lecture documentarisante», dans JeanCharles Lyant et Roger Odin (éd.), Cinémas et réalités, Saint-Etienne, CIIEREC/ Université de Saint-Etienne, I984, pp. 263278.

5 Guy Gauthier, dans Le Documentaire, un autre cinéma (Paris, Nathan, I995, p. 57 et sq.), propose une définition des sous-genres qui mêlent documentaire et fiction. Le «documentaire-fiction» obéit à un scénario préalable et mobilise des acteurs. Le «documentaire fictif» est un piège à visée éducative qui doit amener le spectateur à s'interroger sur sa croyance en des images données pour réelles: plusieurs films de Peter Watkins et quelques films de Chris Marker, en particulier Film $8 \mathrm{~mm}$ trouvé dans une ambassade (I974), relèvent de ce genre.

6 Comédienne, chanteuse, actrice, elle vient alors de jouer dans Johnny Banco de Yves Allégret (I968), dans La Prisonnière de Henri-Georges Clouzot (I969) et dans Sobre gustos y colores (I969) de Juan Battle Planas sur un scénario de Vautier.

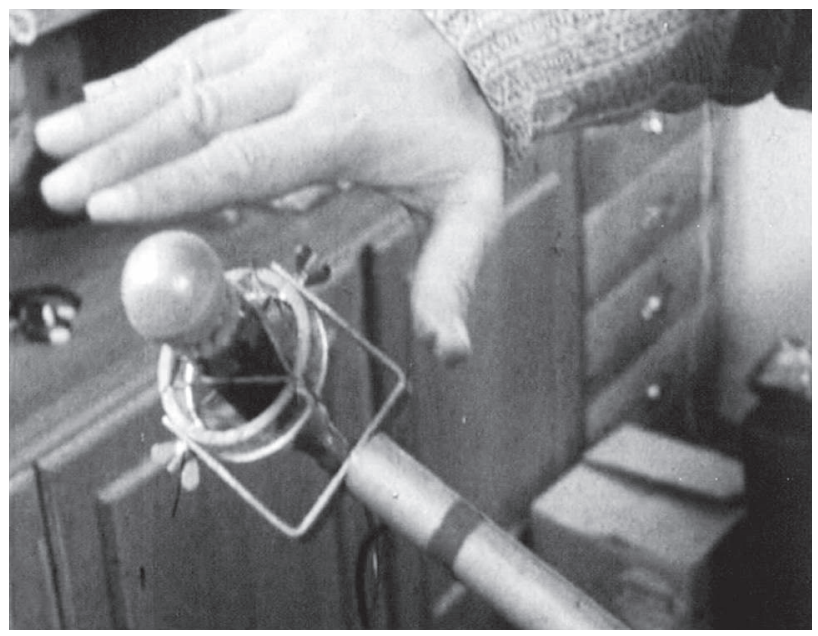

rassemble deux films tournés en collaboration avec Bruno Muel: La Caravelle (4 min) et Techniquement si simple (Io min). Dans le premier, une jeune institutrice en charge d'enfants algériens réfugiés en Tunisie rapporte sa relation compliquée avec un garçon traumatisé par un raid de l'aviation française, qui imagine que la Caravelle qui assure la liaison Paris-Tunis va bombarder son école. Dans le second, un ancien coopérant raconte avec bonhommie comment il répandait des mines dans les champs à l'époque de la guerre d'Algérie. Le statut générique de ces deux films est ambigu: à première vue, ils obéissent tous deux à l'esthétique du «cinéma direct» ${ }^{3}$. Le premier en particulier est constitué d'une seule prise qui commence par l'image de la main de l'ingénieur son en train de tester son micro. Ces deux films mobilisent certes la «lecture documentarisante», pour reprendre les termes de Roger Odin ${ }^{4}$, mais le spectateur a en réalité affaire à une forme hybride, entre «documentaire-fiction» et «documentaire fictif» ${ }^{5}$ : le rôle de l'institutrice est tenu par Elisabeth Wiener ${ }^{6}$ et celui du coopérant par un acteur anonyme. Dans les deux cas, le propos est défini à l'avance, même si une marge d'interprétation est laissée à l'acteur.

Avoir 20 ans dans les Aurès constituait déjà une première expérience de mélange générique. Même s'il s'agit clairement d’une reconstitution 
doublement rétrospective - il s'agit de filmer en 1972 une diégèse située en I96ı et la première partie se construit sur une série de flash-backs -, le film comporte une dimension documentaire affirmée. D’abord, le récit des aventures du «commando breton» s'inspire d'un essai littéraire ${ }^{7}$ et se base sur une série de témoignages, le générique déroulant initial annonçant:

«Cette chronique filmée est en quelque sorte le condensé de 800 heures de témoignage auprès de 600 appelés ou rappelés de la guerre d'Algérie ${ }^{8}$. [...] La véracité de chaque séquence peut être certifiée par un minimum de cinq témoins.»

Ensuite, le tournage est conçu comme un «happening» ${ }^{9}$ : les acteurs sont invités à s'approprier leur rôle et à improviser à partir d'une mise en situation, la caméra portée à l'épaule tentant à plusieurs reprises de capter au vol des bribes d'action. Suivant cette logique, les acteurs sont régulièrement interrogés face caméra, selon un dispositif relevant des actualités filmées. Cette incertitude générique peut faire songer à Punishment Park de Peter Watkins, sorti un an auparavant. Enfin, le film de Vautier orchestre un va-et-vient permanent entre images «mises en scène» et documents «réels », en particulier des images documentaires tirées d'Algérie en flammes (1957) et des extraits de communiqués radiophoniques
7 Dans Désert à l'aube (Paris, Minuit, I960), Noël Favrelière raconte sa désertion en compagnie d'un prisonnier en 1956. RAS d'Yves Boisset (1973), sur un sujet proche d'Avoir 20 ans dans les Aurès, est quant à lui adapté du livre homonyme de Roland Perrot (Paris, Martineau, I970). La comparaison entre les deux films permet de cerner toute l'inventivité générique de Vautier par rapport à une fiction plus classique.

8 Le terme constitue à l'époque une provocation: il faut attendre 1999 pour que l'Etat français reconnaisse la situation de «guerre» en Algérie.

9 René Vautier, Caméra citoyenne, op. cit., p. 228.

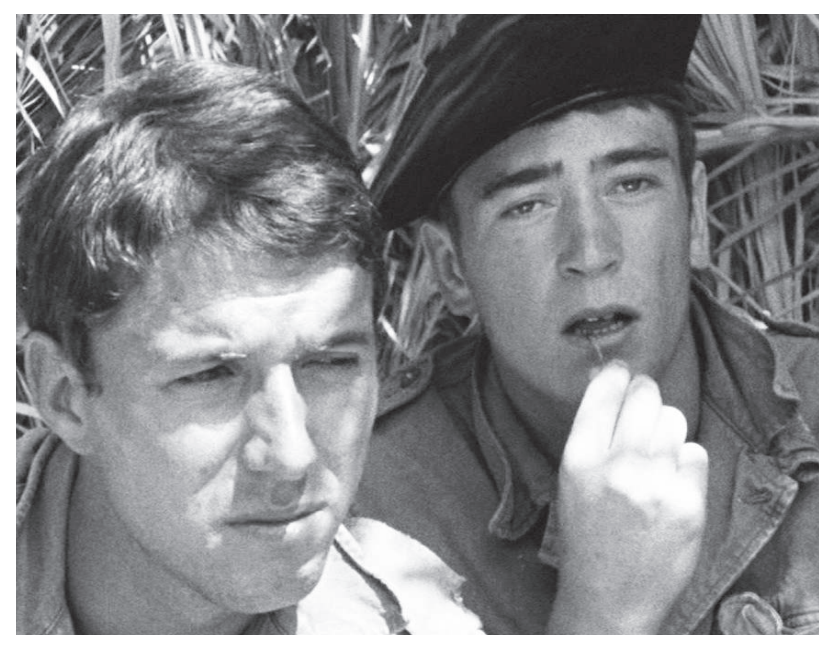

Avoir 20 ans dans les Aurès (1972):

témoignage face caméra 
10 En I977, Vautier réalise Carte blanche à Gilles Servat, film consacré à sa profession de chanteur et à son activisme au sein de l'Union Démocratique Bretonne.
11 Il est publié intégralement dans Caméra citoyenne (op. cit., pp. I09-II7), assorti de ce commentaire: «Pour refléter l'optique des autres, ceux qui avaient choisi de se taire, j’avais écrit en $1956 \mathrm{Le}$ Remords, que je souhaitais tourner en court métrage. [...] J'ai dû attendre I7 ans avant de pouvoir le tourner. [...] Lorsque j’ai présenté ce texte à divers amis acteurs, pour leur demander s'ils accepteraient de tourner le rôle du réalisateur, tous se sont défilés.» (René Vautier, $\mathrm{Ca}$ méra citoyenne, op. cit., p. Iog et p. iı6). relatifs au putsch des généraux en avril 196ı. Faut-il voir dans cette ambiguïté générique une forme de manipulation du spectateur? En aucun cas: nul illusionnisme ici. Cette forme permet au contraire de faire exister rétrospectivement le film manquant sur la guerre d'Algérie.

La Folle de Toujane se situe lui aussi dans un entre-deux entre fiction et documentaire, mais de façon moins marquée. Le film mobilise des acteurs non professionnels qui jouent en quelque sorte leur propre rôle: paysans bretons pauvres (dont les parents de Nicole Le Garrec et leurs voisins) et habitants du village de Toujane en Tunisie. Gilles Servat est un chanteur breton, connu notamment pour la chanson La Blanche Hermine (1972) qui est reprise dans le film, de même que d'autres titres de son répertoire. Il apparaît dans La Folle de Toujane en tant que tel, le générique donnant à voir un récital du chanteur, puis en tant que personnage, incarnant le jeune Roger qui vient d'achever son service militaire ${ }^{10}$. Micheline Welter, dont c'est la première expérience en tant qu'actrice, mènera par la suite une carrière de comédienne. Comme dans Avoir 20 ans dans les Aurès, La Folle de Toujane convoque de nombreuses images d'archive, dont plusieurs ont été tournées par Vautier: répressions en Tunisie en 1953, bombardement du village tunisien de Sakiet Sidi Youssef le 8 février 1958, visite de De Gaulle à Alger en mai I958, dessins et témoignages d'enfants issus du film J'ai 8 ans (I96I, coréalisé par René Vautier, Olga Baïdar-Poliakoff et Yann Le Masson), images de réfugiés et du maquis issues d'Algérie en flammes, plans des défilés célébrant l'indépendance issus de Peuple en marche (1963), et enfin manifestations et luttes ouvrières en Bretagne au début des années I970.

\section{Le Remords, un film dans le film}

La référence au réel apparaît notamment dans Le Remords, une sorte d'intermède enchâssé dans le film qui est l'occasion pour Vautier d'engager une réflexion méta-discursive sur l'engagement cinématographique. Ce sketch de douze minutes dont le scénario remonte à $1956^{11}$ occupe une place centrale dans La Folle de Toujane et connaît d'ailleurs dès 1974 une exploitation à part en tant que court métrage. Il se situe environ à la moitié du film qu'il coupe en deux parties, la première étant consacrée à la séparation du couple Roger-Gwen puis au séjour du jeune homme en Tunisie, et la seconde à la lutte de Roger aux côtés des maquisards algériens puis des paysans bretons. René Vautier y joue avec une jubila- 
tion visible le rôle d'un cinéaste commercial de gauche, qu'aucun acteur n’a voulu incarner. Celui-ci rend visite à sa maîtresse, Gwen, afin de lui raconter qu'il vient d'être le témoin d'un acte raciste: il n'a rien fait pour s'y opposer, mais il y consacrera, un jour sans doute, un "grand film». Cet intermède est central en termes d'économie dramaturgique, certes, mais il constitue surtout une sorte de condensé signifiant du film dans son intégralité: il fonctionne comme un microcosme qui met en abyme La Folle de Toujane.

D'abord, Le Remords permet de conférer davantage de densité au personnage de la jeune fille et permet de creuser l'idée - aux accents néorousseauistes - d'un pervertissement lié à la vie parisienne. Cette idée apparait dès l'incipit de La Folle de Toujane. Commentant les amours entre Roger et Gwen, fille de fermiers de retour dans sa Bretagne natale pendant les vacances, le narrateur note en voix over: «A Paris, ils n'avaient pas l'air de l'avoir éteinte.» En effet, la jeune fille se caractérise par sa liberté et sa sensualité: elle s'émerveille des beautés de la nature, elle se baigne nue dans l'océan ou encore elle invite Roger à faire l'amour dans une ferme abandonnée. Cette spontanéité est soulignée par de nombreux regards caméra mutins. Cependant, Gwen a déjà choisi de faire sa vie à Paris: «La
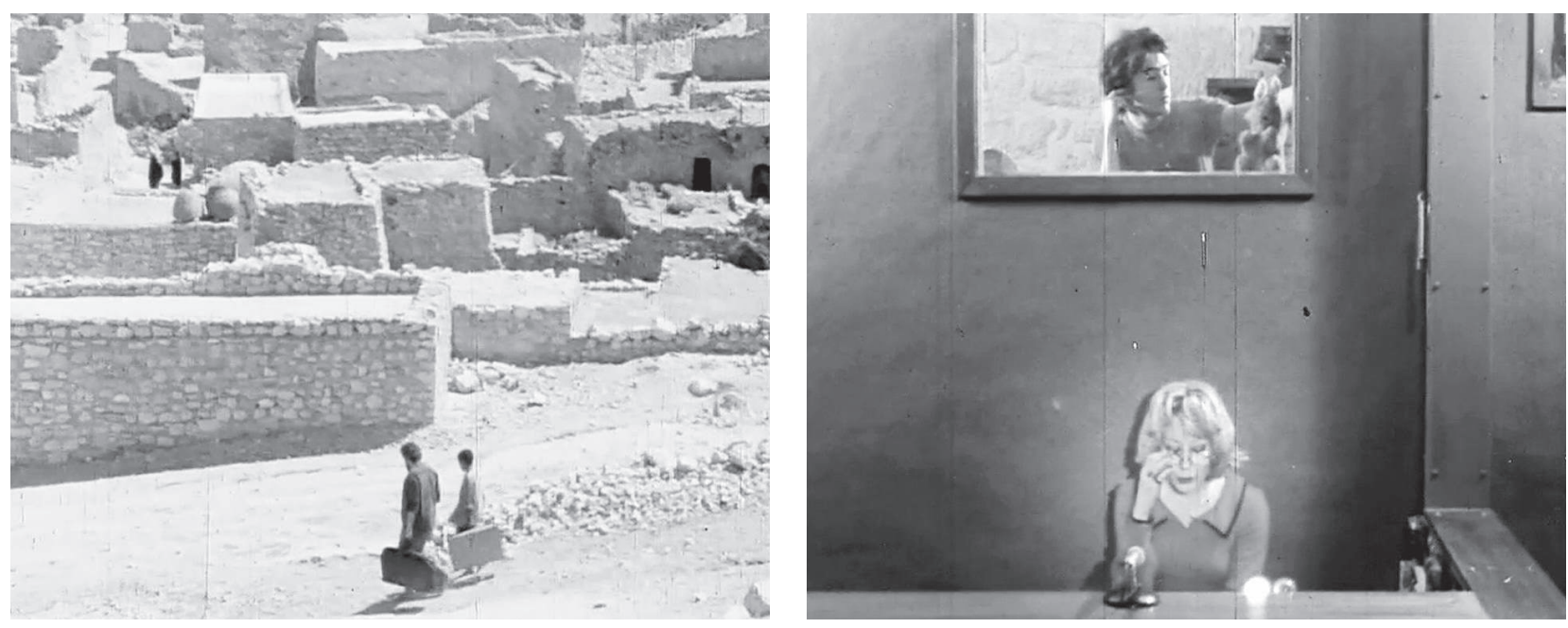
Bretagne c'est foutu... J'veux me faire connaître, y'a qu'à Paris qu'on peut faire ça... J'veux faire du journalisme, de la radio!» Les vacances finies, elle rentre à la capitale, tandis que Roger est nommé en Tunisie. Son séjour à Toujane est mis en scène à travers un montage alterné systématique qui l'inscrit en parallèle avec l'évolution de la carrière de Gwen.

Dès son retour, celle-ci trouve un poste de speakerine grâce à un cinéaste «protecteur» joué par René Vautier. Dans une séquence filmée en plan séquence, on voit celui-ci téléphoner à un ami producteur de télévision avec un air mielleux:

«Ta dernière émission est fulgurante, et cette manière de faire passer la pub derrière, extraordinaire... J'espère que ça te rapporte... Une voiture pour ta femme? Comme ça tu risques rien, et tu l'as bien mérité... J'ai devant moi une charmante demoiselle...»

La contrepartie sexuelle à cette obtention d'emploi est explicite: il caresse la hanche de Gwen et lui adresse des regards éloquents tout en discutant avec son interlocuteur. Une fois le téléphone raccroché, la séquence se conclut par cet échange: «- Je ne sais pas comment vous remercier. - On trouvera bien une façon!» Le monde professionnel, en particulier celui de l'audiovisuel, est clairement montré comme une forme de prostitution. Gwen commence alors son travail de speakerine, les plans répétitifs soulignant son aliénation: surcadrage, sujétion hiérarchique (l’ingénieur son apparaît bizarrement dans une fenêtre située au-dessus de Gwen) et uniformisation (ses chemises arborent les mêmes couleurs que le pull de l'ingénieur son). Les rares variations dans ces plans traduisent la désillusion et le laisser-aller qui s’installent progressivement: rhume, bigoudis, bouteille de whisky...

L'intermède Le Remords amène justement le spectateur à nuancer son jugement à l'égard de la jeune fille - et permet au film centré sur un garçon rebelle et une fille conformiste d'échapper au risque de misogynie. Lors de cette rencontre avec son "protecteur», qui apparaît pour la deuxième fois dans le film, Gwen semble tiraillée entre l'indignation et la compromission résignée. Le dialogue, qui révèle la lâcheté du cinéaste face à un acte raciste, est peut-être l'occasion d'une prise de conscience: lorsqu'on la retrouve à son travail, le cadrage plus rapproché n'induit plus l'idée de sujétion et elle porte une blouse à fleurs fantaisiste renvoyant à la mode hippie. Au même moment, Roger s'engage auprès du FLN algérien. Mais au cours de la conversation avec son amant, elle reconnaît qu'elle n'ima- 
gine pas céder son appartement pour financer un film militant: «Je suis aussi minable que toi.» La conclusion de La Folle de Toujane est profondément pessimiste. Gwen est finalement nommée à la télévision grâce à une «promotion canapé», ce que laisse comprendre une question de son collègue ingénieur du son: «C’est vrai que t'es sortie avec le patron?» Gwen adresse alors un sourire moqueur à la caméra: «T’inquiète pas, j’l'aurai mon manteau de vison!» Dans une ultime apparition consécutive à la mort de Roger, elle semble avoir définitivement sombré dans l'alcool.

\section{Racisme ordinaire et violence coloniale}

Le Remords apparaît par ailleurs comme une dénonciation de la violence policière. Le cinéaste parisien vient se confier à Gwen après avoir été témoin d'un passage à tabac particulièrement violent: un Algérien s'adresse à deux agents pour leur demander un renseignement, ils le rouent de coups et le laissent pour mort dans le caniveau. Cette «bavure» peut rappeler le court métrage Les Ajoncs (1970), dans lequel un gendarme pris de rage détruit la carriole du travailleur algérien qui s'est improvisé marchand de fleurs. Mais par-delà la dénonciation de la violence policière, si l'on tient compte du fait que le scénario du Remords a été écrit en I956 et que la diégèse est censée se passer en 1958, il est clair que Vautier renvoie, par-delà ce récit, à la guerre d'Algérie et à ses conséquences en France. Cette petite scène fonctionne en particulier comme une allusion au massacre des Algériens à Paris le i7 octobre i96ı : un an avant le tournage de La Folle de Toujane, entre décembre 1972 et janvier I973, Vautier a mené une grève de la faim pour protester contre la censure du documentaire de Jacques Panijel, Octobre à Paris (I96I), consacré à ce massacre.

Le racisme ordinaire de la police française apparaît ainsi comme un cas particulier de la violence coloniale, qui est le véritable sujet de La Folle de Toujane. La dizaine de séquences rapides consacrées à Gwen et son métier de speakerine permettent de suivre en filigrane les luttes de la Tunisie et de l’Algérie pour leur indépendance. Sont mentionnés en particulier: l'accord de coopération France-Tunisie signé par Mendès-France et Bourguiba (1954), l’indépendance de la Tunisie (20 mars 1956), le bombardement du village tunisien de Sakiet Sidi Youssef (8 février I958), le putsch d'Alger et l'arrivée de De Gaulle au pouvoir (mai I958). Par-delà ce rappel des événements historiques, la violence coloniale transparaît à travers l'expérience de Roger en Tunisie. A son arrivée, la déclaration 
12 Georges Perec, Les Choses (Paris, Julliard, I965; rééd. Paris, ıo/ı8, 2009), p. II9, p. I22 et p. I3I.

13 Cette situation entre deux feux rappelle d'une certaine manière la propre situation de Vautier en 1958, que le cinéaste évoque dans Guerre aux images en Algérie (I985). Les services secrets français provoquent la méfiance du FLN à l'égard de Vautier, suspecté de «dévier le sens de la révolution algérienne dans un sens communiste», ce qui amènera son emprisonnement pendant vingt-cinq mois: «Je me suis retrouvé dans une prison algérienne alors qu'on me recherchait côté français pour me mettre dans une prison française pour aide au FLN. C'est une position inconfortable d'être ainsi recherché de tous les côtés.» de l'inspecteur de l'Education nationale («Vous serez à l'avant-poste de la culture française...») est vite balayée par un sentiment d’inutilité. Cette expérience rappelle la deuxième partie du roman de Perec Les Choses (1965), dans laquelle l'auteur transpose le séjour effectué en compagnie de son épouse à Sfax en i96o en qualité de coopérant, et dont voici quelques extraits qui entrent parfaitement en résonnance avec le film de Vautier:

«Leur solitude était totale. Sfax était une ville opaque. [...] Ils n’éprouvaient ni joie, ni tristesse, ni même ennui, mais il pouvait leur arriver de se demander s'ils existaient encore, s'ils existaient vraiment. [...] Monde sans souvenirs, sans mémoire. Du temps passa encore, des jours et des semaines désertiques, qui ne comptaient pas.» ${ }^{12}$

A la différence du roman de Perec qui se situe après l’indépendance de la Tunisie et dont les anti-héros ignorent complètement la guerre d’Algérie voisine, La Folle de Toujane réfère explicitement l'absurdité de la situation de Roger à celle de la situation coloniale.

Vautier dénonce en particulier «les livres aberrants et inadaptés» qui amènent Roger à évoquer «nos ancêtres les Gaulois» devant de jeunes Tunisiens perplexes. Le jeune homme décide alors de faire la classe dans la rue et commence à apprendre l'arabe, ce qui n'est guère du goût de l'inspecteur qui rappelle en ces termes le rôle de l'enseignement: «Nous les formons pour aller en France. L'industrie française a besoin d'eux.» Quand il rentre à Toujane après une suspension de deux mois, Roger découvre le corps de deux de ses élèves tués pendant une opération de «pacification» pour avoir écrit le mot «liberté» sur le mur de l'école. Il tente alors de s’intégrer à la population, mais les villageois le renvoient à sa solitude, comme l'indique la narration en voix over: «Traître à la France, étranger au village $»^{13}$. Le choix de l'engagement concret contre le colonialisme est provoqué par l'épisode centré sur Saloua, la «folle de Toujane» qui donne son nom au film. Cette ancienne élève de Roger se marie avec un Algérien en juillet i957 et part en Algérie. Elle revient quelques années plus tard, après avoir été torturée et avoir vu ses enfants tués par l'armée française. Folle de douleur, elle finit par se suicider. Roger quitte alors Toujane, devient instituteur dans un centre de regroupement des Aurès puis infirmier dans un maquis tenu par l'ALN. Suite aux accords d'Evian, il rentre en Bretagne: «Il a compris qu'on ne peut lutter que chez soi.»

Le film fait ainsi le lien entre la colonisation des pays du Maghreb et la violence semi-coloniale en Bretagne, évoquant l'exode rural (nom- 
breuses fermes à vendre), les petits paysans ruinés et expropriés (le père de Gwen se suicide au même moment que Saloua) et l'implantation d'un terrain militaire sur la terre des parents de Roger, réminiscence de la lutte sur le plateau du Larzac dans la première moitié des années i970. Roger meurt d'ailleurs en traversant un chant de mines, scène qui rappelle le nécessaire déminage en Algérie évoqué dans Peuple en marche ou la description des enfants estropiés dans Techniquement si simple. Cette mort n'est pas vaine cependant: le film se conclut sur des images des luttes indépendantistes et ouvrières en Bretagne après Mai 68. De nombreuses critiques publiées dans la presse au moment de la sortie du film s'interrogent d'ailleurs sur le bien-fondé de cette convergence des luttes, à l’image de cet article paru dans L'Humanité qui évoque «l'amalgame encore un peu trop rapide entre les drapeaux tunisien, algérien et breton $»^{14}$. Un seul critique s'enthousiasme pour la mise en exergue du problème breton:

«La Folle de Toujane fera sans doute date. [...] Il consacre la naissance d'un cinéma breton, comme il y a un cinéma algérien ou chilien. Pour la première fois dans le cinéma, toutes les séquences se situant en Bretagne sont en breton et sous-titrées en français. ${ }^{15}$

\section{Censure et auto-censure}

Si La Folle de Toujane opère une synthèse entre les deux grands combats de Vautier, l'Algérie et la Bretagne, le film permet aussi l'émergence d'un nouveau thème qui domine la seconde partie de la carrière du cinéaste: la question de la censure et de l'autocensure. Cette question apparaît pleinement dans l'intermède Le Remords. Vautier y campe le prototype du cinéaste bien-pensant, sorte de «nouveau beauf» à la Cabu avec foulard et cigare, qui permet d'engager la réflexion sur les paradoxes de l'engagement artistique.

Au moment où il est le témoin d'un acte odieux, ce personnage est incapable d'action, peut-être par une sorte de déformation professionnelle qui fait de lui «un pur regard»:

«J'ai été obligé de regarder ça parce que je ne pouvais pas bouger. [...] Je suis plus une caméra qu'un homme. [...] J'ai enregistré tout ça, je pouvais pas démarrer. [...] Ça va se développer comme la pellicule.»

L'absence de réaction est alors compensée par un discours logorrhéique, le monologue virant au soliloque, et par un vague projet: «Après
14 Albert Cervoni, «D’Afrique en Bretagne», L'Humanité, 7 septembre I974.

15 Ernest Cedic, «La Folle de Toujane», Le Quotidien de Paris, 6 juin 1974. 

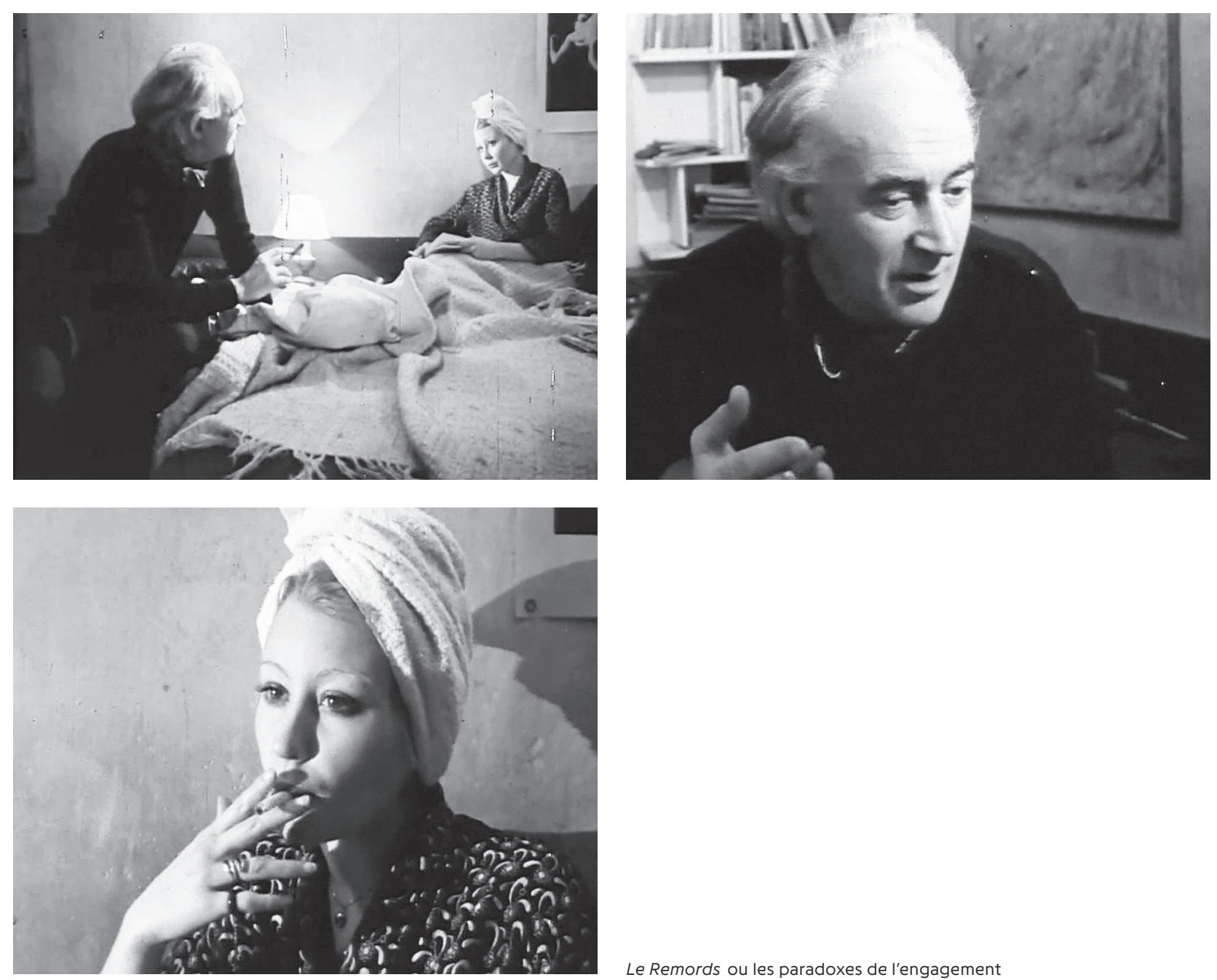

Le Remords ou les paradoxes de l'engagement

je ferai un film, une œuvre, une dénonciation, un cri.» La mauvaise foi de ce discours éclate grâce aux réponses rapides et précises de Gwen:

«- Dans combien de temps? - Je sais pas, dans cinq ans [...]. - Quand ça n’aura plus aucune portée? - [...] Je suis pas un reporter d'actualités, moi.

- Tu fais du film historique. - [...] Non, je fais des films sur des choses qui 
se passent, mais les choses de ce genre, pour nous, il faut le temps de les digérer. - Tu sais ce que ça donne les choses trop digérées? - Ça fait de la merde, ah ça c'est malin.»

Ainsi, au fil de la discussion, l'intention politique perd de son mordant et le projet final est réduit à une interrogation solipsiste sur les atermoiements de l'engagement:

«Maintenant je vais rentrer, je vais prendre quelques notes. [...] Dans cinq ans ce sera un accouchement, je ferai un film formidable. [...] Ce film, je l'appellerai L'Infamie. Les flics en prendront plein la gueule. [...] Mais ils s'arrangeraient encore pour faire interdire le film. [...] Non je ferai un film sur le remords de n’avoir rien fait, j’appellerai ça Le Remords, c'est pas mal comme titre, non?»

Ce personnage fictif permet à Vautier d'opposer la démission de certains cinéastes au courage de ceux qui mènent un travail de contreinformation quel qu'en soit le prix. Déjà, Avoir 20 ans dans les Aurès entendait restituer le point de vue de la base en opposant à l'Histoire événementielle la petite histoire du «Commando breton», comme l'indique le générique déroulant: «A l'heure où les généraux, les politiciens et les agents secrets donnent chacun leur version de ce que fut la guerre d'Algérie, nous avons pensé qu'il était utile de refléter l'état d'esprit des appelés.» Dès le générique, La Folle de Toujane engage lui aussi la réflexion sur ce terrain, en parodiant l'ORTF, Vautier déclarant en voix over: «Le rôle de la télévision nationale est d'illustrer les phrases marquantes de la pensée gouvernementale.» Le texte déroulant énonce immédiatement après: «Le réalisateur, l'acteur, les chansons et la maison de production de ce film sont pratiquement interdits d'antenne par le directeur de l'ORTF Rennes.» La séquence Le Remords pointe a contrario les dangers de l'autocensure pratiquée par les cinéastes qui craignent de se couper des producteurs ou des critiques et in fine de se retrouver déclassés économiquement ou socialement.

Comme Avoir 20 ans dans les Aurès, La Folle de Toujane entend racheter rétrospectivement la rareté des films français dénonçant la guerre d'Algérie au moment des faits: étant donné le contrôle sur la production audiovisuelle exercé par l'Etat ${ }^{16}$, les images de rares cinéastes comme Paul Carpita, Gilles Chalon et Philippe Durand, ainsi que celles de Pierre Clément et de René Vautier, font exception. Celui-ci s’interroge à plusieurs reprises sur l'incapacité de ses collègues à monter des projets
16 Voir Sébastien Denis, Le Cinéma et la guerre d'Algérie. La propagande à l'écran, Paris, Nouveau Monde éditions, 2009; Benjamin Stora, Imaginaires de la guerre. Les images de la guerre d'Algérie et du Vietnam, Paris, La Découverte, 1997. 
17 René Vautier, Caméra citoyenne, op. cit., p. 27.

18 Vautier revient sur cette histoire dans Caméra citoyenne (op. cit., p. 96) et dans «Entretien avec René Vautier», Cahiers du cinéma, n ${ }^{\circ}$ 5 1 , octobre 200I, pp. I4-I9. sur ce thème, en particulier dans Guerre aux images en Algérie (1985). Au début de ce film, la coréalisatrice Elisabeth Frasson-Marin rapporte une enquête menée par Michèle Manceaux pour L'Express en I960 auprès des cinéastes de l'époque, à qui la journaliste posait cette question: «Si la censure n'existait pas, est-ce que vous auriez envie de tourner un film sur la guerre d’Algérie?» Plusieurs répondent qu’ils sont en train de développer un scénario sur ce thème, dont Alain Resnais et Pierre Kast. Aucun n'aboutira. Elisabeth Frasson-Marin mentionne la conclusion qu'en tire Georges Sadoul: «On peut faire de grands discours sur la liberté d'expression, mais on ne peut pas en France faire un film contre l'avis des banques. Et la guerre d'Algérie est aussi une affaire de banquiers.» Vautier revient longuement sur les démêlés de Resnais avec la censure dans Caméra citoyenne:

«L'effet le plus pernicieux n’a pas été la suspension temporaire des films Les Statues meurent aussi et Nuit et brouillard. Ça a été l'avertissement donné à un jeune réalisateur de talent, en début de carrière: si tu ne plies pas, on te casse. [...] Il se rendra vite compte que parler en images de la guerre d'Algérie peut entraîner beaucoup d'ennuis; et il choisit de s'en aller en Allemagne, tourner un film apolitique qu'il faudra, dit-il, «regarder comme une statue». Ce sera L'Année dernière à Marienbad. C'est en voyant ce film [...] que je me suis dit: la censure a gagné. ${ }^{17}$

Toujours dans Guerre aux images en Algérie, Vautier rappelle l'expérience de Philippe de Broca (sans le nommer), lequel part en Algérie pour le compte du SCA (Service cinématographique des armées) avec le projet de réaliser en sous-main «un film sur la vérité en Algérie». Choqué par les exactions dont il est le témoin, son comportement laisse penser à ses supérieurs hiérarchiques qu'il risque de changer de camp et de diffuser des images gênantes. La police fait alors pression sur lui et les deux parties arrivent à un compromis, que Vautier résume en ces termes: «Si vous me réformez et m’envoyez en France, je m'engage à vous envoyer toute la pellicule sans la faire développer.» Broca justifie alors auprès d'une amie son renoncement à faire un film de dénonciation: «C'est les documents contre ma peau et je préfère ma peau.» ${ }^{18}$ Dans le même film, Vautier évoque à l'inverse le sort de deux cinéastes qui ont filmé aux côtés des Algériens: Cécile de Cugis, condamnée à deux ans de prison pour un film sur les réfugiés en Tunisie, et Pierre Clément, condamné à dix ans de prison pour sa présence dans les maquis algériens. 


\section{Monologues politiques: des prétextes à l'inaction}

Le long monologue que constitue Le Remords entre en écho avec d'autres monologues à teneur politique permettant de manifester la mauvaise conscience et/ou la mauvaise foi d'un personnage masculin face à un personnage féminin généralement muet. On pense au Petit soldat, le film de Godard sur la guerre d'Algérie tourné en i960 et censuré jusqu'en ig63, et en particulier à la longue scène finale entre Bruno (Michel Subor) et Veronika (Anna Karina). Celle-ci affirme: «Les Français ont tort. [...] Ils perdront la guerre.» Le jeune homme se lance alors dans une véritable logorrhée et expose la complexité - faut-il dire la confusion? - de ses idées politiques:

«Je suis très fier d'être français, mais en même temps je suis contre le nationalisme. On défend des idées, on ne défend pas des territoires. J'aime la France parce que j'aime Jean Du Bellay et Louis Aragon. [...] J'aime l’Amérique parce que j’aime les voitures américaines et je n'aime pas les Arabes parce que je n’aime pas le désert ni le colonel Laurence, encore moins la Méditerranée et Albert Camus.»

Face aux protestations suscitées par ce film, Godard tente de se justifier en ces termes:

«Moi, j’ai parlé des choses qui me concernaient, en tant que Parisien de 1960, non incorporéà un parti. Ce qui me concernait, c'était le problème de la guerre et ses répercussions morales. J’ai donc montré un type qui se pose plein de problèmes. Il ne sait pas les résoudre, mais les poser, même avec l'esprit confus, c'est déjà tenter de les résoudre.» ${ }^{19}$

On retrouve un monologue semblable dans le segment «Claude Ridder» réalisé par Resnais pour le film collectif Loin du Vietnam (1967) orchestré par Chris Marker: un personnage fictif d'intellectuel (Bernard Fresson) est chargé d'écrire un texte provietnamien. Il ne peut s'y résoudre et se livre à une longue autocritique portant sur sa bonne conscience militante, face à un personnage de femme allongée et muette, mais au regard lourd de reproches. Ce segment se situe exactement dans le même registre que celui réalisé par Godard pour le même film et intitulé «Camera Eye». Godard, qui apparaît à l'écran et parle en son nom propre, refuse lui aussi d'endosser le rôle du témoin dénonciateur, car parler au nom des combattants vietnamiens serait une imposture:

«C'est difficile de parler des bombes alors qu'on ne les reçoit pas sur la tête. [...] Plutôt que d'essayer d'envahir le Vietnam par une générosité qui force les choses, au contraire il faut laisser le Vietnam nous envahir.»
19 «Entretien avec Godard», Cahiers du cinéma, $\mathrm{n}^{\circ} \mathrm{I} 38$, décembre 1962 , repris dans Jean-Luc Godard par Jean-Luc Godard, t. I, Paris, Cahiers du cinéma, I998, p. 220. 
Le Petit soldat de Godard (1961) et «Claude Ridder » de Resnais dans Loin du Vietnam (1967): monologues politiques
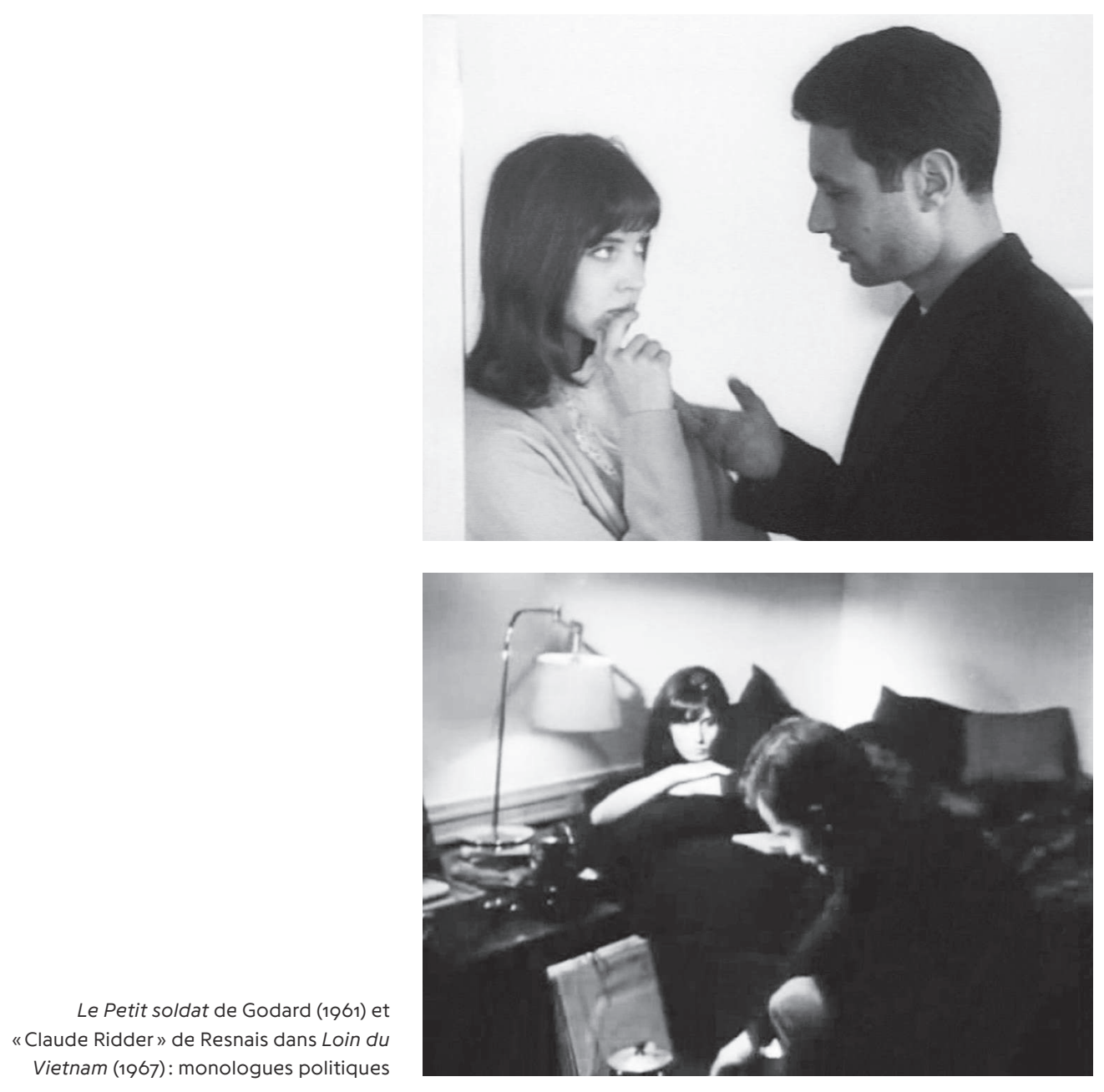

Dans cette perspective, il est passionnant de lire le dialogue entre Godard et Vautier qui confrontent leur conception de l'engagement lors d'une rencontre organisée par «Les écrans citoyens» en 2002. Godard revient sur son sketch pour Loin du Vietnam:

«Je ne voyais pas ce qu'on pouvait faire: mettre des images du Vietnam me paraissait douteux pour quelqu'un qui habite la France, qui en est 
loin. Le film s'appelait du reste Loin du Vietnam. La moindre des choses était donc de montrer qu'on était loin du Vietnam.» ${ }^{20}$

Face à ces scrupules qui peuvent paraître excessifs dans un contexte d'urgence politique, Vautier répond:

«On fait des films sur ce que l'on connaît bien, mais on a aussi la possibilité de faire des films sur ce qu'on essaie de comprendre. Pendant la guerre d'Algérie, il y a eu un grand silence du cinéma français, comme il y a eu un grand silence sur le plan du colonialisme. Là, ne fallait-il pas essayer de faire quelque chose, même si on n'avait pas le droit de le faire?» ${ }^{21}$

Face à ces quelques exemples d'autojustification, où la parole ne parvient pas vraiment à combler l'absence d'action, Vautier montre dans La Folle de Toujane que pour lui, contrairement à son double négatif du Remords, faire un film c'est réellement: «faire quelque chose».
20 Nicole Brenez, David Faroult, Michael Temple, James Williams, Michael Witt, Jean-Luc Godard, Documents, Paris, Centre Pompidou, 2006, p. 399.

21 Ibid. 